\title{
Image Interpreter Tool: An ArcGIS Tool for Estimating Vegetation Cover From High-Resolution Imagery
}

\section{By T. Scott Schrader and Michael C. Duniway}

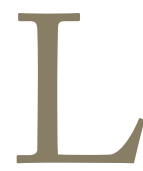

and managers need increased temporal and spatial resolution of rangeland assessment and monitoring data. However, with flat or declining land management and monitoring agency budgets, such increases in sampling intensity are unlikely unless new methods can be developed that capture data of key rangeland indicators at a lower cost. Remote sensing techniques have shown promise for collecting plant community composition and ground cover data efficiently. ${ }^{1-3}$ However, many image analysis techniques require software and expertise not always available to field offices (e.g., Laliberte et al. 20103). Collecting data through image interpretation approaches often is more feasible for many agencies. ${ }^{4}$ For such methods to be applied successfully in assessment and monitoring programs, the image interpretation data collection needs to be done in a repeatable fashion, by users of many backgrounds, in a streamlined and efficient workflow.

Image Interpreter Tool (IIT) is a series of ArcGIS 9.3 tools and workflow procedures that have been developed to meet this need. The tool and procedures were designed to streamline: 1) the calibration of image interpretation users and 2) the collection of vegetation and ground-cover types for a study site or project. IIT is distributed as a customized ArcMap document or template with nothing to install, and is compact enough to be used on portable storage devices such as USB thumb drives. IIT can be used by people with little or no GIS experience and reduces recording errors by providing an automated system for attributing data files.

IIT mimics point-intercept field-sampling methods (such as those developed by Herrick and colleagues 5 ) using remotely sensed data, "virtual" points along transects, and a simple and intuitive interface-to-estimate cover. Three main cover categories are used: noncanopy (rock, litter, soil, and lichens), herbaceous cover (grass and forbs), and woody canopy (subshrub, shrub, tree, and succulent). Additionally, users can toggle between true color and color-infrared versions of the imagery (assuming four-band imagery is used as a source) with a simple click of a button on the interface. IIT is easy to learn and is designed to facilitate multiple users producing consistent results. IIT is divided into two modules: 1) an observer training and calibration module that includes quality assurance and quality control procedures and 2) a data collection module.

\section{Observer Training and Calibration Module}

The quality assurance and quality control procedures in the observer calibration module help ensure that first-time and experienced users are familiar with the interface and can make general vegetation and ground-cover type identifications consistently and accurately. The calibration module was designed to transfer the knowledge of experts (i.e., individuals both familiar with identifying ground and vegetation cover types and trained in image interpretation) to less experienced image interpretation users, and then provide a proficiency assessment to assure they are adequately trained.

In image interpretation, users need to understand how key features such as tone, color, texture, pattern, context, shape, and size differ among cover classes. ${ }^{6}$ This understanding of key differences between cover types is transferred from expert to nonexpert users via a written decision key and, most importantly, a high-resolution nadir image with accompanying expert-classified points used as a training dataset. The decision keys provide a logical path of deduction to determine cover type and to allow the user to consistently classify each point in a plot based on observations and descriptive characteristics for each type. The expert-classified point layer is used both to train users in correct classifications on a point-by-point basis and then subsequently test the users' understanding.

\section{Observer Training (Quality Assurance)}

The expert-classified points are evenly divided into two groups - training and testing points—by randomly assigning every other transect as either a training or testing transect. 


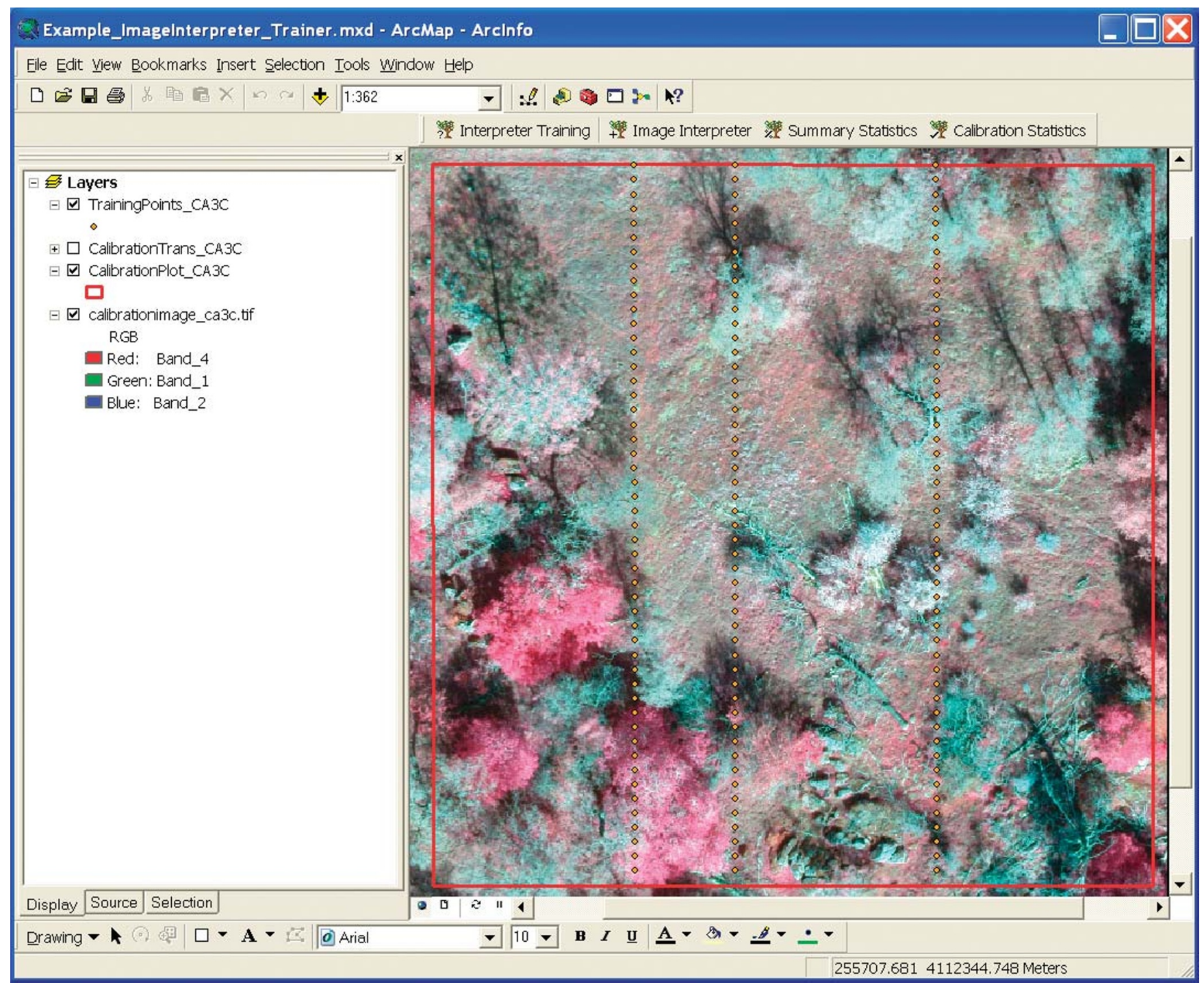

Figure 1. Color-infrared imagery (3-cm pixels) of a training and calibration site in California. In color-infrared imagery, verdant vegetation appears in shades of red of varying intensity. The main tool bar of the Image Interpreter Tool (IIT) is visible as a series of four buttons located directly above the displayed image. The extent of training and calibration plot $(50 \times 50 \mathrm{~m})$ is identified by the red polygon. In this example, there are three transects of sample points (orange dots) visible that were randomly selected to be used as training dataset; the remaining transects of points (not shown) will be used in subsequent "calibration testing" phase of the tool.

A user starts the trainer by selecting the "Interpreter Training" button from the main tool bar (Fig. 1). The tool zooms to the first training location and presents a simple interface (Fig. 2) where the user can make cover selections by clicking on the intended cover-type button provided in the interface. Incorrect selections cause the selected covertype button to be highlighted in red, providing instant feedback to the user. Correct selections cause the tool to move to the next training location. The instant feedback is designed to train users to correctly identify the specific vegetation and ground cover represented in the training dataset, as well as provide training in the basic functions of the tool itself. The user continues viewing and selecting appropriate cover types for subsequent training locations. The navigation buttons that are visible in the interface are purposefully disabled in training mode to force a user to examine each sample location in sequence for proper training.

\section{Observer Testing (Quality Control)}

When the user has completed all of the calibration training points, they are tested to evaluate their ability to distinguish among the different vegetation and ground-cover types through a calibration-testing procedure. The testing procedure begins by selecting the "Image Interpretation" button from the main tool bar while evaluating the testing points. In calibration testing mode, the tool performs the same tasks as in training mode, but without immediate feedback for incorrect classification of points. As in the trainer, the IIT zooms to the first of the areas selected for evaluation and presents a simple interface of cover type selections. The 


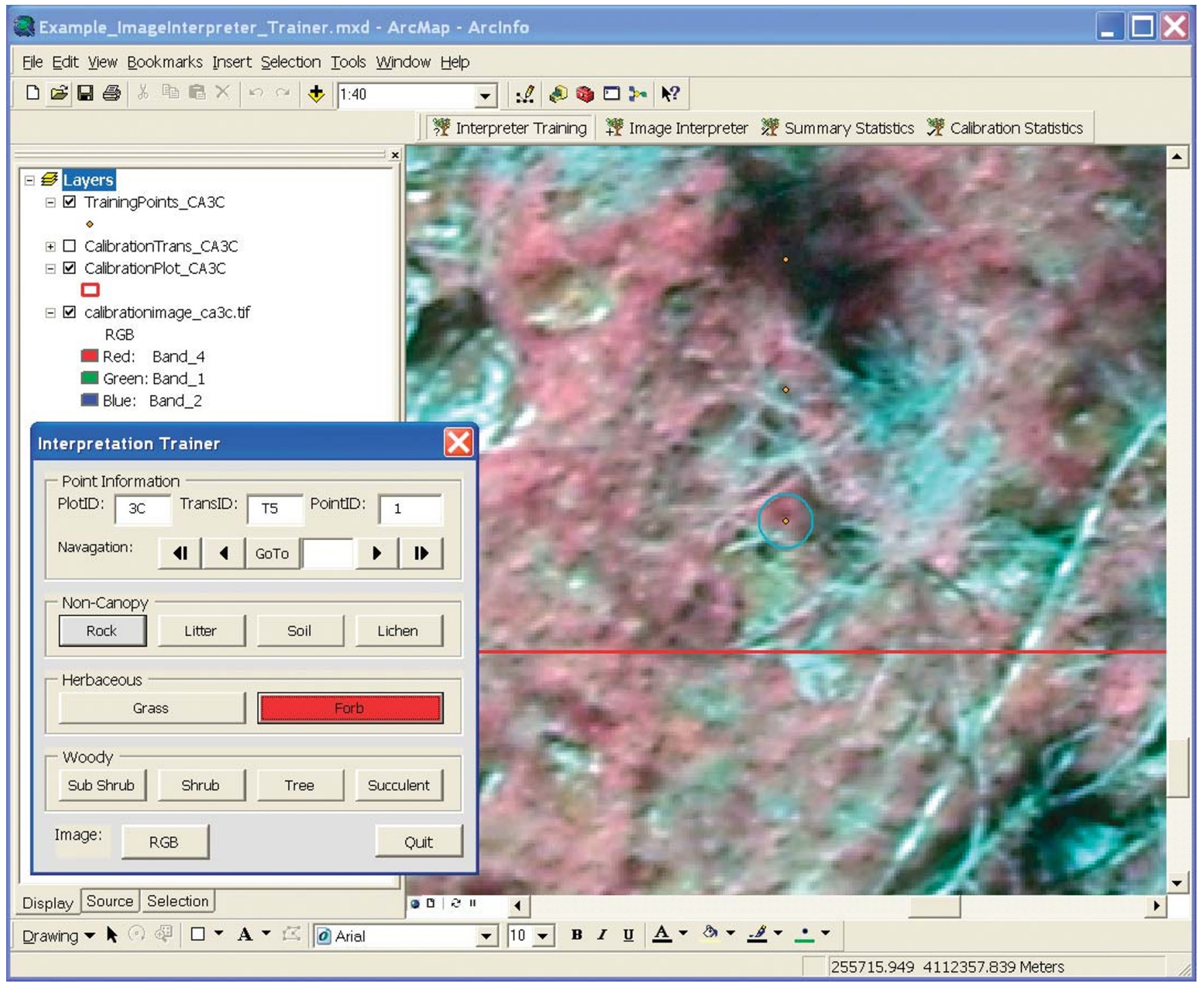

Figure 2. The training interface presented when the user selects "Image Trainer" from main tool bar. The tool automatically zooms to the first training location. User makes selections by clicking on the intended cover type button provided in the interface. Incorrect identifications cause the selected cover type button to be highlighted in red, providing instant feedback to the user. Correct selections cause the tool to cycle to the next training location.

user makes cover type identifications by selecting the intended button and the tool proceeds to the next sequential calibration testing location after automatically attributing the value.

After all calibration testing locations have been identified, the user is prompted to save "Summary Statistics" and a window with the vegetation and ground cover metrics is presented (Fig. 3). Results include percent cover for each generalized cover category as well as for each specific cover type. Percent cover is calculated automatically by plot and by transects. User results can be exported to a common spreadsheet format and compared to other users' evaluations or to an expert evaluated calibration testing dataset by using the "calibration statistics" button (Fig. 4). Users can import any existing summary statistics file to calculate differences in cover for comparison, and the tool reports and saves the differences between the two statistical summaries by site and transect. Test results can be evaluated to determine if users are sufficiently calibrated to the expert dataset and to discover where improvements in identification might be needed. For example, users could be required to achieve a less than 5\% difference in percent cover compared to the expert classifications before they can proceed to data collection on an actual study site.

\section{Data Collection Module}

Once a user is calibrated sufficiently, they can select the "Image Interpreter" button to begin classifying points on an actual study site. The IIT zooms to the first sample location and presents the familiar interface with a few more options (Fig. 5). In data collection mode, a user can navigate to any specific sample location. If the location has been identified previously, the user can select "keep type" to migrate to the next sequential location or reattribute the location among 


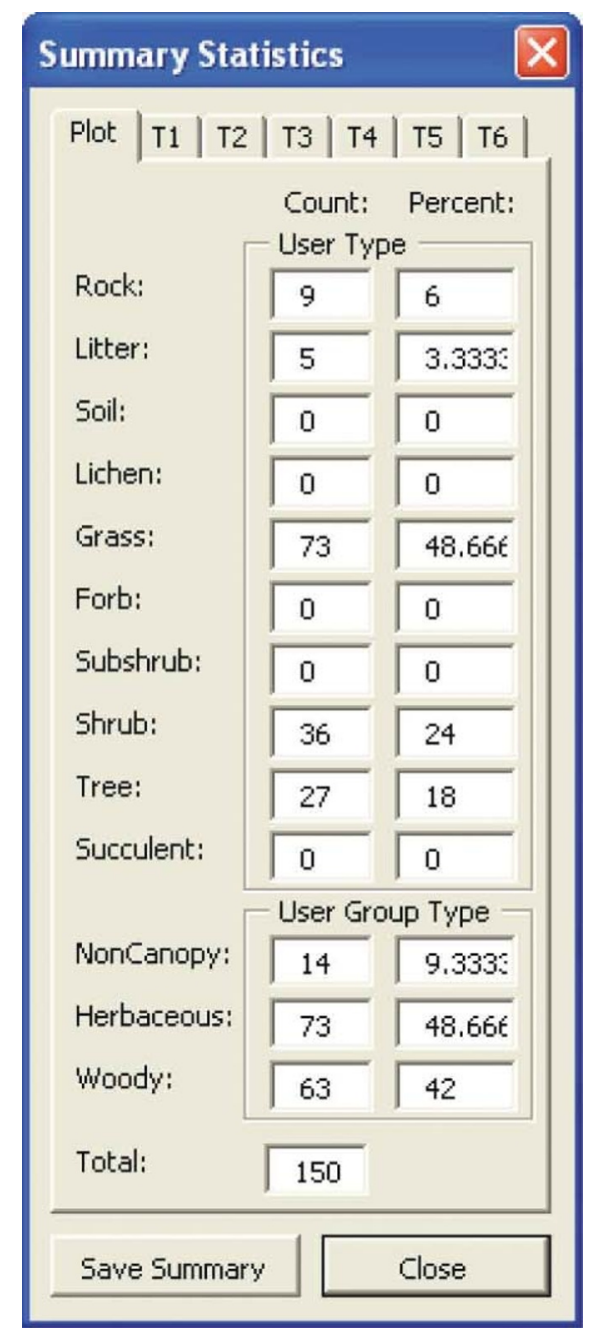

Figure 3. Summary Statistics window that is presented automatically when a user completes an evaluation of calibration testing points or when a user selects the "Summary Statistics" button from the main tool bar. Percent cover for each generalized cover category and for each specific cover type is calculated automatically by plot, as well as by individual transect.

the available cover types. This allows a user to re-examine locations as many times as necessary to be certain of the proper identification.

The comment dropdown feature of the interface allows users to add additional information for a specific location. This is useful if a user is uncertain of the cover type identification and would like to note the reason for the confusion so the location may be re-evaluated at a later time. This dropdown is populated with user-entered values that once entered, become available in the comment dropdown at any subsequent location. This eliminates the need for users to repeatedly enter the same comment information; once it is entered, it is available for use via the dropdown.

The "detail on/off" toggle button available in the data collection module is used to allow a user to enter more detailed information related to a specific sample location. For example, if a user discovers that several locations contain

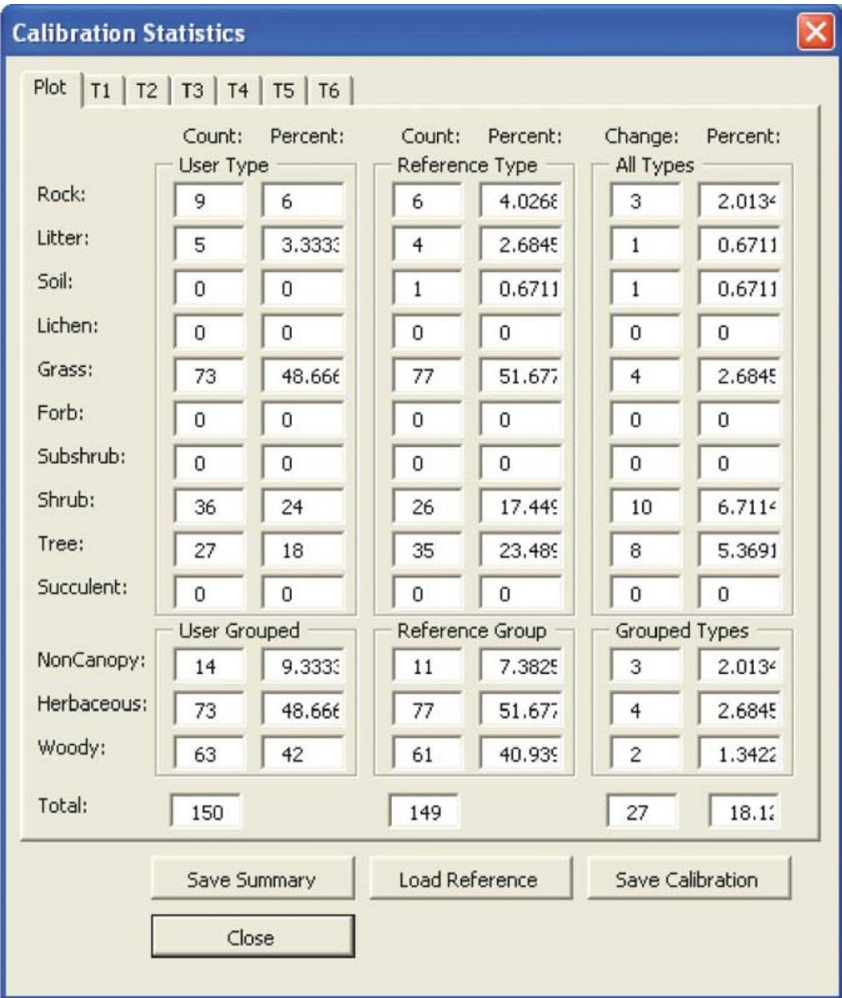

Figure 4. Calibration Statistics form presented when a user selects the "Calibration Statistics" button from the main tool bar. The user can import any existing summary statistics file and calculate differences in cover for comparison. The tool reports and saves the difference between the two statistical summaries by site and transect.

a shrub that can be identified to the species level within the imagery, he/she can toggle the detail button, make the shrub selection, and a second dropdown field is presented allowing the user to enter the specific information (Fig. 6). Similar to the comments feature, anything entered within these detailed dropdown fields become available at any subsequent location. The detail function can be turned off for locations that do not warrant additional information or to streamline work flow in support of various project objectives. There is no need to save data manually; the IIT automatically saves the data each time a point is classified. When all points in the data collection plot have been classified, the IIT presents plot cover statistics.

\section{Current Limitations and Future Developments}

The process and tools developed cannot overcome all of the potential problems associated with image interpretation. ${ }^{6}$ Data collection with IIT only can be successful if the imagery used is of sufficient resolution and quality to distinguish classes of interest. ${ }^{7}$ Additionally, great care must be taken to ensure the calibration datasets are accurately classified and representative of the study site. It is important to have consistent and exclusive definitions for each cover type to help users avoid confusion among types. Ideally, experts should classify all the points in the calibration site many 


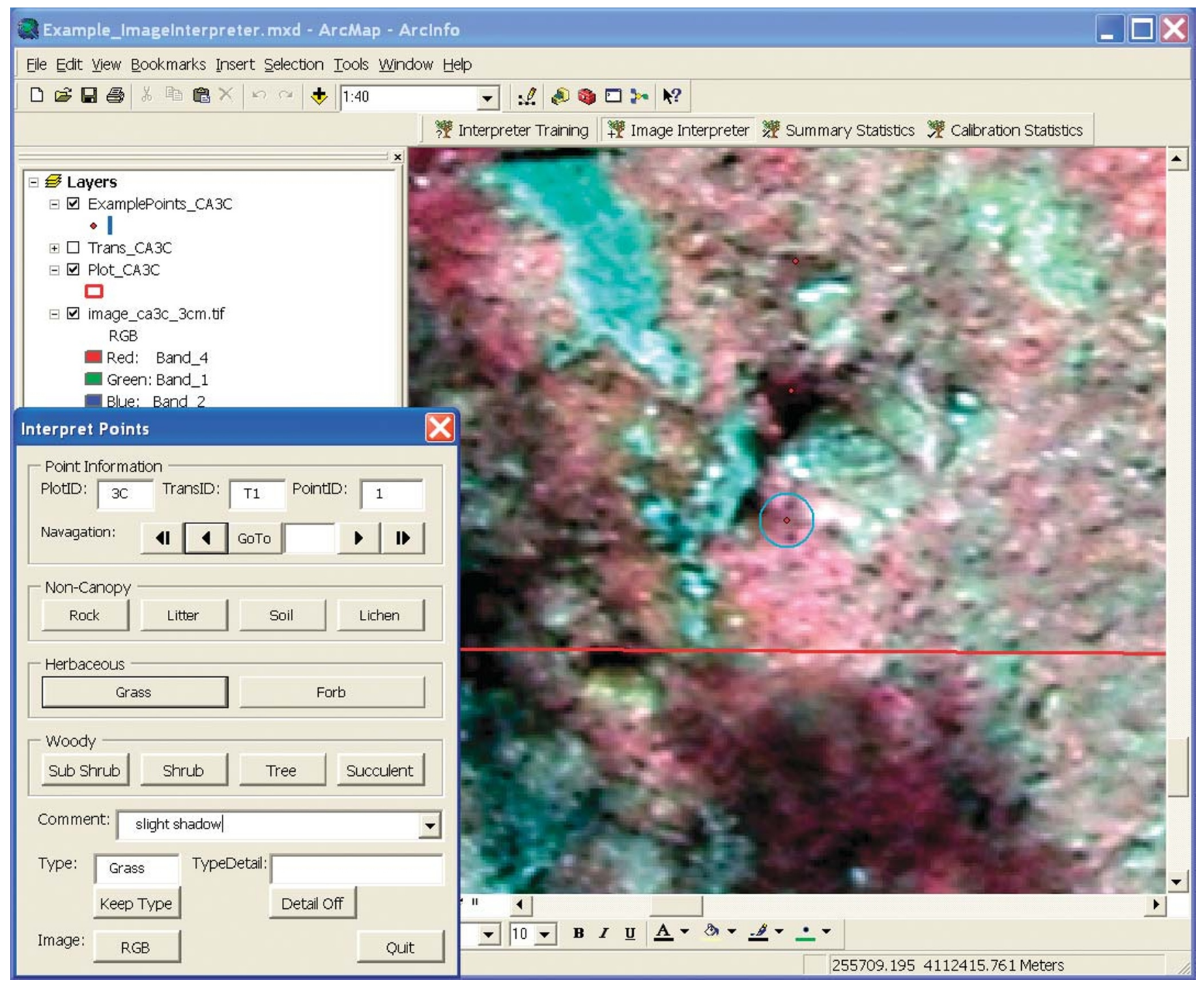

Figure 5. Data collection interface presented to a user by selecting the "Image Interpreter" button from the main tool bar. Additional features are available to allow a user to record general comments for each evaluated location within this interface. Navigation buttons and a "keep type" button are provided to allow a user to reidentify cover types as many times as necessary to ensure an accurate evaluation of the site.

times to create the benchmark values. We currently are developing procedures that use the relationship between the point classifications and image information to evaluate if the points were classified in a consistent manner. Such a test could be used as an indicator of the accuracy of calibration data sets and observer data collection.

Currently IIT, requires significant set up of data layers by an experienced Geographic Information System (GIS) user. GIS skills are required to create the needed plot, transect, and sample point datasets. The tool expects spatial data to be in a specific order for the tool to function correctly, and it has limitations in the types of imagery that can be included (i.e., 4-band images in either ERDAS Imagine [.img] or TIFF [.tif] format). Also, the tool's zoom levels are hardprogrammed into the interface; therefore, imagery used with these tools should be very high resolution $(<4 \mathrm{~cm})$.
We currently are developing an additional interface and tools to allow less experienced GIS users automatically to perform set up functions, such as defining plot, transects, and sample locations for a study site. We also are creating administrative tools to set specific attributes for the tool; for example, zoom levels for a project that would be appropriate for various resolution imagery, randomization of point locations, or setting the number of expected transects for statistics generation. Additional spatial data types will be incorporated for use as spatial inputs such as geodatabase feature datasets. A new version of this tool will be developed utilizing "Plug-ins" for ArcGIS 10 compatibility. Lastly, an "Enterprise GIS" version of the tools will be created and distributed as a web service and will be accessible through a web browser without the need for a traditional ArcGIS installation. 


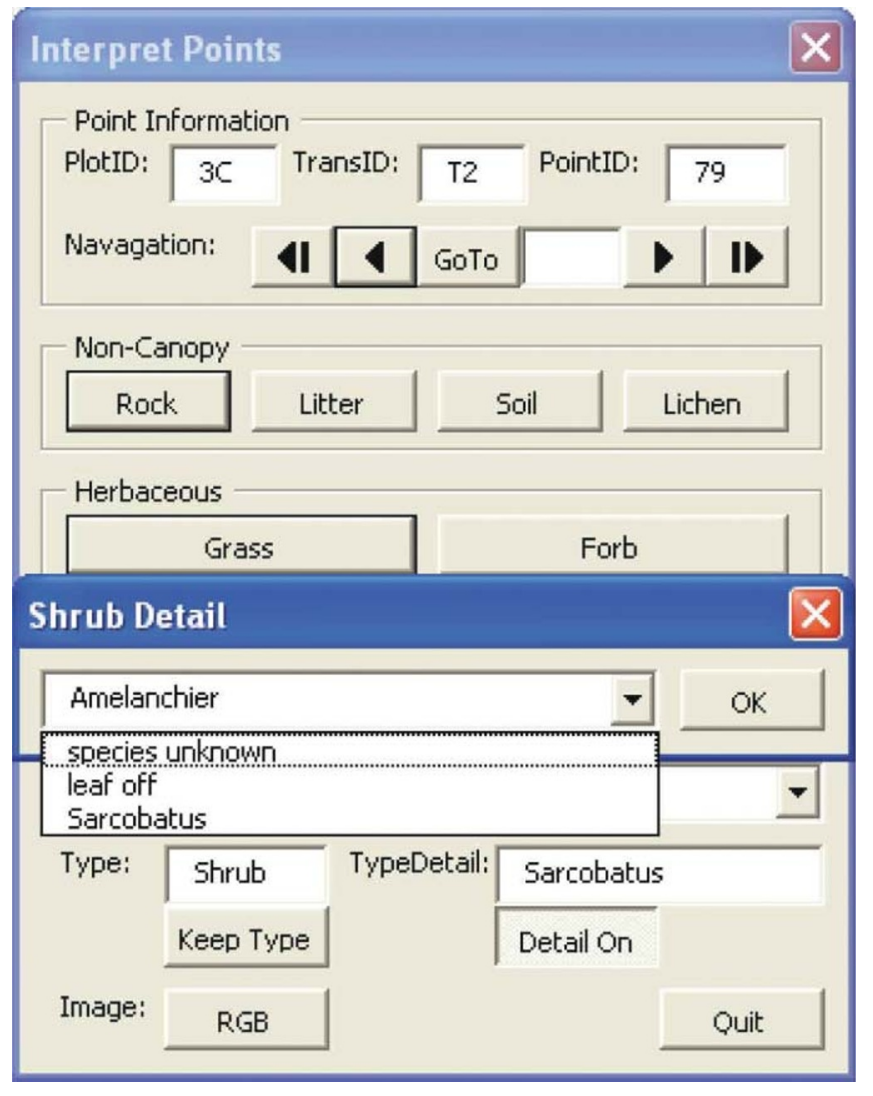

Figure 6. Detailed types combo box presented to a user by utilizing the "Detail on/off" toggle button at a specific sample location. The detailed combo box dynamically records any attribute entered therein and the values become available to the user for selection at any subsequent location.

\section{Conclusion}

The IIT can be used to augment field sampling efforts with nonfield-generated data by simulating point intercept field methods using high-resolution imagery and virtual transects of sample points. With IIT, the act of attributing the sampled point to generalized vegetation and ground-cover types is streamlined, requiring the user to have little or no GIS experience. The training and testing calibration procedures help reduce incorrect identifications and, most importantly, transfers expert knowledge of vegetation and ground cover characteristics to less-informed users. This knowledge transfer system allows remote sensing data on key rangeland indicators to be collected by users at remote locations and potentially can reduce the workload of local land management and monitoring agencies.

\section{References}

1. Boоth, D. T., and P. T. Tueller. 2003. Rangeland monitoring using remote sensing. Arid Land Research and Management 17:455-467.

2. Hunt, E. R., J. H. Everitt, J. C. Ritchie, M. S. Moran, D. T. Booth, G. L. Anderson, P. E. Clark, and M. S. Seyfried. 2003. Applications and research using remote sensing for rangeland management. Photogrammetric Engineering and Remote Sensing 69:675-693.

3. Laliberte, A. S., J. E. Herrick, A. Rango, and C. Winters. 2010. Acquisition, orthorectification, and object-based classification of unmanned aerial vehicle (UAV) imagery for rangeland monitoring. Photogrammetric Engineering and Remote Sensing 76:661-672.

4. Воотн, D. T., And S. E. Cox. 2008. Image-based monitoring to measure ecological change in rangeland. Frontiers in Ecology and the Environment 6:185-190.

5. Herrick, J. E., J. W. Van Zee, K. M. Havstad, L. M. Burkett, And W. G. Whitford. 2005. Monitoring manual for grassland, shrubland and savanna ecosystems. USDA-ARS Jornada Experimental Range. Las Cruces, NM, USA: University of Arizona Press. Vol. 1, Quick Start. 36 p.

6. Morgan, J. L., S. E. Gergel, and N. C. Coops. 2010. Aerial photography: a rapidly evolving tool for ecological management. Bioscience 60:47-59.

7. Воотн, D. T., S. E. Cox, And D. E. Johnson. 2005. Detectionthreshold calibration and other factors influencing digital measurements of ground cover. Rangeland Ecology \&' Management 58:598-604.

Authors are GIS Specialist, Schrader@nmsu.edu (Schrader); and Research Soil Scientist (Duniway), USDA-ARS Jornada Experimental Range, Las Cruces, NM 88003-8003, USA. 